\title{
Mathematical Formulation For Computation Of Optimal Number Of Sensor Nodes For Effective Coverage In Wireless Sensor Networks
}

\section{J. Samuel Manoharan ( $\nabla$ drjsm1530@ieee.org)}

Sir Isaac Newton College of Engineering \& Technology https://orcid.org/0000-0001-8281-3993

\section{Research Article}

Keywords: Wireless sensor networks, Connected graph, Coverage, Random deployment, Redundancy coverage and Sensor nodes.

Posted Date: February 15th, 2022

DOI: https://doi.org/10.21203/rs.3.rs-854550/v1

License: (a) (i) This work is licensed under a Creative Commons Attribution 4.0 International License. Read Full License 


\title{
Mathematical Formulation for Computation of Optimal number of sensor nodes for Effective Coverage in Wireless Sensor Networks
}

\author{
Dr. J. Samuel Manoharan \\ Professor/Dept. of ECE, Sir Isaac Newton College of Engineering \& Technology, Nagapattinam \\ - 611102, South India \\ drjsm1530@ieee.org
}

\begin{abstract}
Wireless sensor networks have been the major stake holders in almost all types of wireless communication standards and applications in the recent decade. Their ability to be deployed almost anywhere on the globe make them indispensable. However, in the research of the Wireless sensor networks (WSN), trouble in coverage is one of the fundamental problems. Coverage computation in a way finds a major role to find out the energetic amount of sensor nodes required for sensing the monitoring region. Initially the investigator deals the resolving process of maximum flawless ratio of coverage for three-sensor nodes. Then by the resultant of work, grants the solution method of the estimated value of quality of coverage and also provides the method in elaborate manner for finding the least amount of sensor nodes for effective coverage of monitoring area. Finally, the technique to find out the redundancy for $n$ communicative nodes has been provided. All the above issues have been mathematically worked out to justify the coverage research issue.
\end{abstract}

Keywords: Wireless sensor networks, Connected graph, Coverage, Random deployment, Redundancy coverage and Sensor nodes.

\section{Introduction}

Wireless sensor network (WSN) is a small group of independent sensor nodes scattered in the space with partial power. Such nodes have the capability to observe the substantial conditions and communicate data from one node to another node at different locations by wireless links. It is only possible if there is a path from each and every node to that monitoring area. Since sensing as well as connectivity is the interrelated features in WSNs. Usually, the connectivity of WSN is considered by a graph in which vertices are match to the sensor nodes and an edge from one vertex to another vertex point out the data communication. If every node 
contains same communication ranges, then the graph turns out to be undirected. Generally, every network can be considered as connected. Consequently, the associated graph is also connected. As we know a graph $\mathrm{G}$ is connected if there exists a path between every pair of its vertices [1].

\subsection{Surrounding Nodes}

The Euclidean distance between any two sensor nodes is less than twice the possible radius is called as the surrounding nodes.

\subsection{Connected Graph}

Assume that the sensor nodes $S_{i} \in V$. There exists a unidirectional edge $\left(S_{1}, S_{2}\right)$ in $E$ if and only if the sensor nodes $S_{1}$ and $S_{2}$ are within the transmission span of each other. Also if the congruence of nodes represented as the number of surrounding nodes then $G=(V, E)$ is called as Connected Graph.

A particular case of connected graph is the unit disk graph that embedded in Euclidean space. The Euclidean space is defined as a path between every two nodes whose Euclidean distance is less than or equal to radius $r=1$. The complete summary on Euclidean distance will be given in the middle of this work.
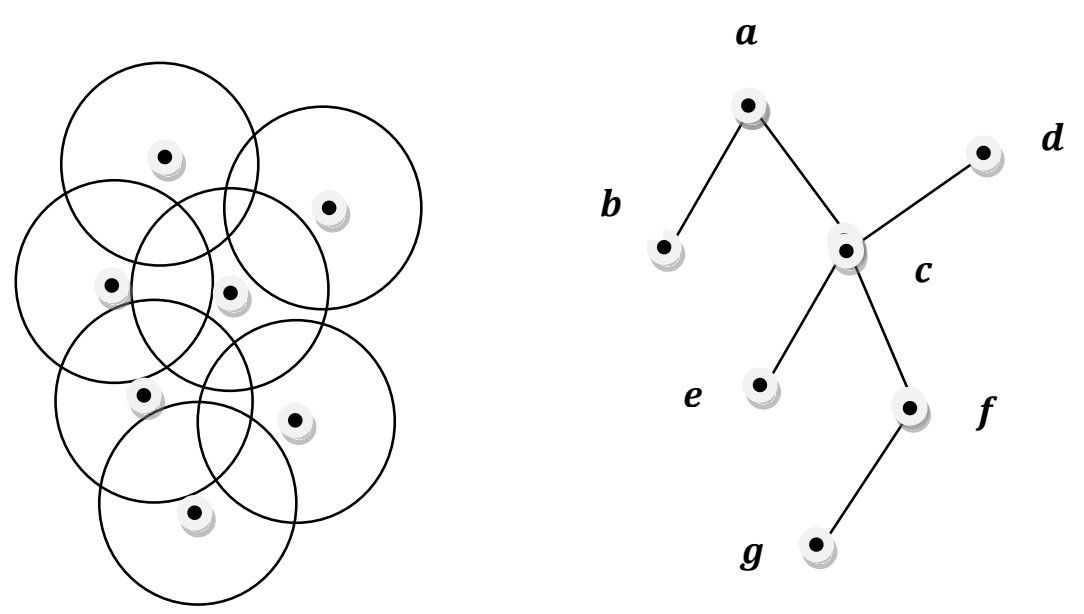

Fig: 1 Representation of Unit Disk Graph $(V, E)$

$$
\boldsymbol{V}=\{\boldsymbol{a}, \boldsymbol{b}, \boldsymbol{c}, \boldsymbol{d}, \boldsymbol{e}, \boldsymbol{f}, \boldsymbol{g}\}, E=\{(\boldsymbol{a}, \boldsymbol{b}),(\boldsymbol{a}, \boldsymbol{c}),(\boldsymbol{c}, \boldsymbol{d}),(\boldsymbol{c}, \boldsymbol{e}),(\boldsymbol{c}, \boldsymbol{f}),(\boldsymbol{f}, \boldsymbol{g})\}
$$




\section{Review of Related Literature}

Consume of energy and quality of coverage are the most important notions in the domain of WSN analysis [2,3]. These two are mostly dependent on the prudence of deployment of the sensor nodes [4]. In random deployment method we cannot predict the accurate spot of the sensor nodes. As a result, there exists abundance of sensor nodes in $S$ with the coverage of degree is k. Recently many experts and researchers were analyzed in this area. Adulyasas et al. [5] proposed the algorithm for improving the efficient coverage of entire observing area. Liao et al. [6] proposed the heuristic approach to balance the energy consumption of network. Sun et al. [7] presented the barrier coverage method with the maximum lifetime of reinforcement. Mini et al. [8] delivered three algorithms namely artificial intelligence algorithm, swarm intelligence and the granule approach for the deployment of sensor nodes in the observing area. Zhao et al. and Hanid et al. $[9,10]$ offered the Voronoi based algorithm for coverage problems. Zeyu Sun et al. [11] proved the effectiveness through the novel method of coverage in wireless sensor network the random deployment. Rasmi Ranjan Patra et al. [12] determined the degree of coverage of an area covered by two or more sensors and they found the lowest degree of coverage. Ming Liu et al. [13] proposed the mathematical replica in the analysis of coverage in WSNs. They had passed an investigational study by simulation method. Xiaole Bai et al. [14] proved the optimality of a existing deployment pattern for achieving both full coverage and 1-connectivity also they found the required nodes needed to offer coverage and connectivity. Xiaole Bai et al. [15] investigated the pattern of deployment that attains four-connectivity and maximum coverage. Tabirca et al. [16] introduced same lower and upper bounds for smallest number of sensors for k-covering. They also improved the previous result of Ammari et al. [17].

\section{Assumptions and Notations}

\subsection{Assumptions}

- The area of deployment of sensor nodes in 2D space.

- All sensor nodes enclose some radius $\mathrm{r}$.

- Each sensor nodes are self-governing to each other.

- The transmission area is greater than or equal to twice the anticipated radius. 
- The radii of sensor fall in with the normal distribution.

\subsection{Notations}

$S$-The monitoring area

$S_{i}$-The sensor nodes

$R_{i}$-The shaded regions

$T_{i}-$ Regular triangles

$\nabla_{i}-$ Sectors

$q$-Coverage role

$\mu$-The sensing radium

$\mu_{\alpha}-$ The expected value of $\mu$

$\sigma^{2}-$ Varience

$Q-$ Coverage quality

$d$ - Euclidean distance

\section{Effective Coverage Probability}

The system coverage of the effective sensor nodes $S_{i}$ deployed randomly in the monitored region $S$ is

$$
P\left(S_{i}, S\right)=\frac{A\left(\sum_{i=1}^{n} S_{i} \cap A(S)\right)}{A(S)}
$$

Also the effective coverage probability is

$$
P_{c}\left(S_{i}, S\right)=\frac{A\left(\sum_{i=1}^{n} S_{i} \cap A(S)\right)}{A\left(\sum_{n=1}^{n} S_{i}\right)}
$$

\subsection{Determination of Effective Coverage Area}




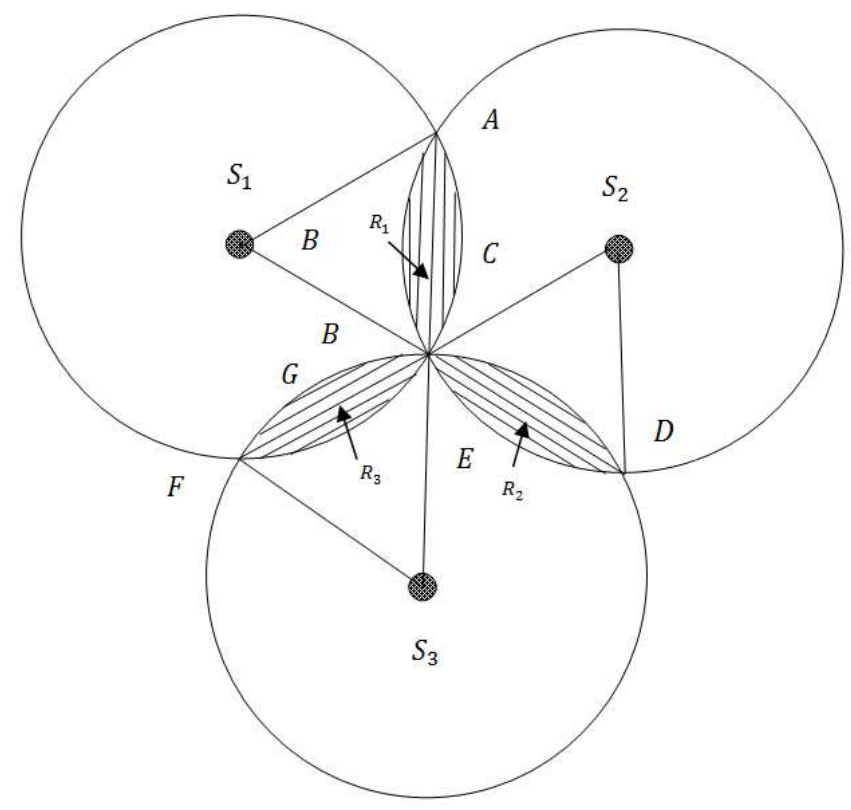

Fig: 2 Flawless Connection of the Three-sensor node

Let us consider the shaded regions are $R_{1}, R_{2}, R_{3}$. Also its corresponding sectors and equiangular triangles are $\nabla_{1}=S_{1} A C B, \nabla_{2}=S_{2} B E D, \nabla_{3}=S_{3} B G F$ and $T_{1}=O_{1} A B, T_{2}=$ $O_{2} B D, T_{3}=O_{3} B F$ respectively. Assume that $S_{1}, S_{2}$ and $S_{3}$ are sensor nodes will join in the intersection spot $B$ due to the maximum coverage area. Here the sectors are one-sixth of the circles consequently each angle are $60^{\circ}$. Since $\triangle S_{1} A B, \triangle S_{2} B D, \triangle S_{3} B F$ are regular then $S_{1} A=A B=B S_{1}=S_{2} B=B D=D S_{2}=S_{3} B=B F=F S_{3}=r$

As it is known,

$$
\begin{aligned}
& \text { The area of sector }=\frac{\theta}{360^{\circ}} X \pi r^{2} \\
& \text { The area of regular triangle }=\frac{\sqrt{3}(\text { side })^{2}}{4}
\end{aligned}
$$

If we draw the line $x=0$ on the graph, we will split the intersection region $R_{1}$ into two equal pieces. 


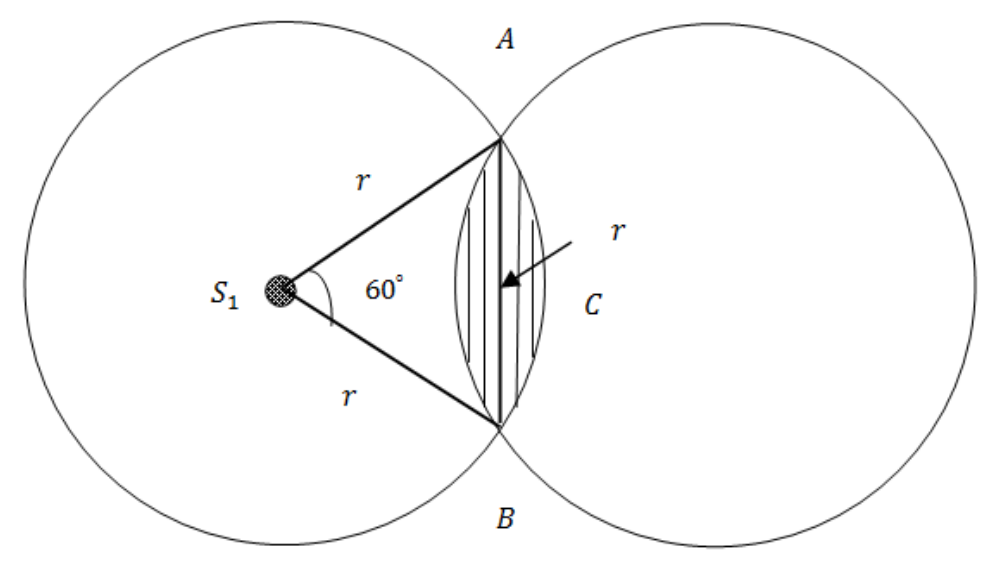

Fig: 3 Representation of two intersect sensors

Since Area $R_{1}=2\left(\right.$ Area $\nabla_{1}-$ Area $\left.T_{1}\right)$

$$
\begin{aligned}
& =2\left(\frac{\pi r^{2}}{6}-\frac{\sqrt{3} r^{2}}{4}\right) \\
& =(2 \pi-3 \sqrt{3}) r^{2} / 6
\end{aligned}
$$

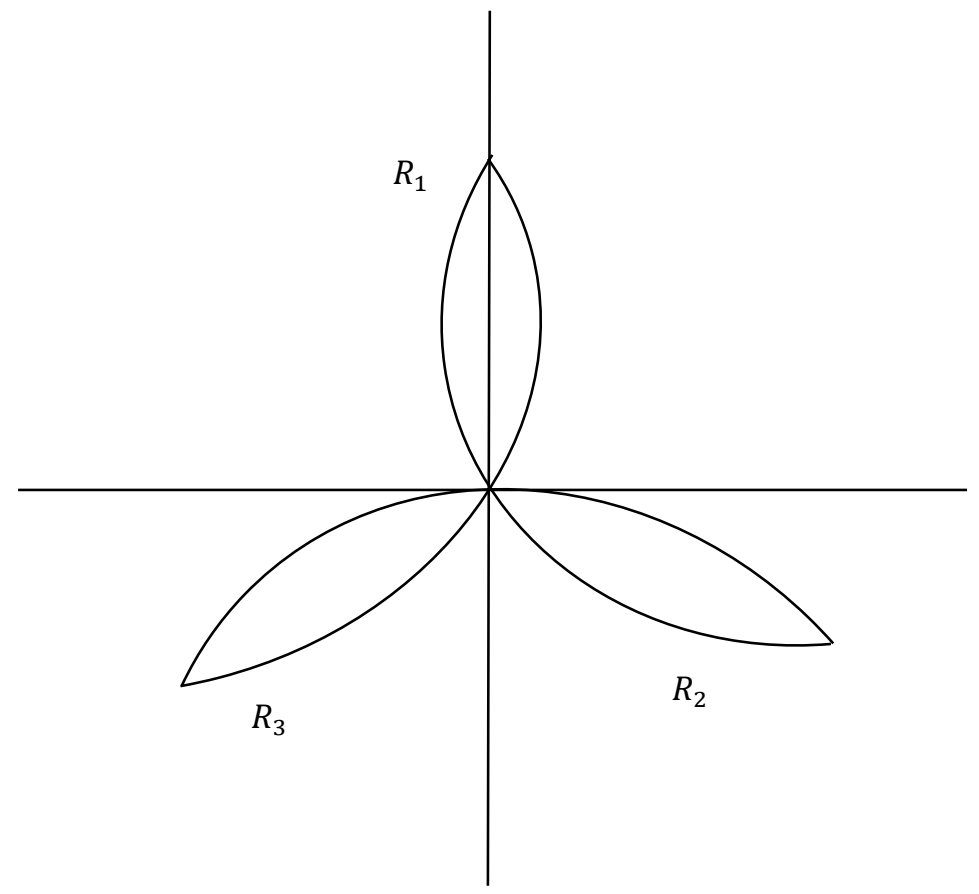

Fig: 4 Representation of pairwise radical lines 
Maximum Coverage Area $\left(\right.$ Cover $\left._{\max }\right)=3\left(\pi r^{2}-\right.$ Area $\left.R_{1}\right)$

$$
\begin{aligned}
\text { Cover } \left._{\max }\right) & =3\left(\pi r^{2}-(2 \pi-3 \sqrt{3}) r^{2} / 6\right) \\
& =\frac{1}{2}\left[4 \pi r^{2}+3 \sqrt{3} r^{2}\right]
\end{aligned}
$$

$$
\begin{aligned}
\text { Eff Cover }_{\text {max }} & =\frac{\text { Cover }_{\text {max }}}{\text { Total area }} \\
& =\frac{\frac{1}{2}(4 \pi+3 \sqrt{3}) r^{2}}{3 \pi r^{2}} \\
& =\frac{4 \pi+3 \sqrt{3}}{6 \pi} \\
& =0.94287 \\
& =94.28 \%
\end{aligned}
$$

\section{Estimation of Expected value of Coverage Quality}

Let $(x, y) \in S$. The existence of minimum one sensor node in $\mathrm{S}$ then it is covered. Assume that the sensor nodes in $S$ are scattered in uniformly at random.

Hence the rate of coverage $q=\frac{\text { Surrounding Area }}{\text { Monitoring Area }}$

$q=\frac{\pi r^{2}}{s_{\text {area }}}$

Since the sensor nodes with the expected radius are distributed normally $\left(\mu_{0}, \sigma^{2}\right)$ in $S$. Then quality of coverage is defined by

$$
\begin{aligned}
Q & =\int_{0}^{2 \mu_{0}} q f(r) d r \\
& =\int_{0}^{2 \mu_{0}} q \frac{1}{\sigma \sqrt{2 \pi}} e^{-\frac{1}{2}\left(\frac{r-\mu_{0}}{\sigma}\right)^{2}} d r \\
& =\int_{0}^{2 \mu_{0}} \frac{\pi r^{2}}{s_{\text {area }}} \frac{1}{\sigma \sqrt{2 \pi}} e^{-\frac{1}{2}\left(\frac{r-\mu_{0}}{\sigma}\right)^{2}} d r \\
& =\sqrt{\frac{\pi}{2}} \frac{1}{S_{\text {area }}} \int_{\frac{-\mu_{0}}{\sigma}}^{+\frac{\mu_{0}}{\sigma}}\left(x \sigma+\mu_{0}\right)^{2} e^{-x^{2} / 2 d x}
\end{aligned}
$$

where $x=\frac{r-\mu_{0}}{\sigma}$ 
After simplifying (1),

$$
\begin{aligned}
& Q=\sqrt{\frac{\pi}{2}} \frac{1}{S_{\text {area }}}\left(\sqrt{2 \pi}\left(\sigma^{2}+\mu_{0}^{2}\right)\right) \\
& Q=\frac{\pi\left(\sigma^{2}+\mu_{0}^{2}\right)}{S_{\text {area }}}
\end{aligned}
$$

Since the $k$-sensor nodes in $S$ are independent to each other then the expected value of coverage quality is $E(Q)=1-\left[1-\frac{\pi\left(\sigma^{2}+\mu_{0}^{2}\right)}{S_{\text {area }}}\right]^{k}$

\section{1 k-degree Coverage}

If k-sensors intersect with each other than the degree of intersection region is known as k-degree coverage.

\section{Establishment of Lowest amount of Sensor nodes}

Assume that the sensor nodes are deployed in $S$. For each point on the edge of $S$, its surrounding area is least. So its probability of being covered is also least value.

$$
\begin{aligned}
& q \leq E(Q) \\
& \frac{\pi r^{2}}{S_{\text {area }}} \leq 1-\left[1-\frac{\pi\left(\sigma^{2}+\mu_{0}^{2}\right)}{S_{\text {area }}}\right]^{n} \\
& 1-\left[1-\frac{\pi\left(\sigma^{2}+\mu_{0}^{2}\right)}{S_{\text {area }}}\right]^{n} \geq \frac{\pi r^{2}}{S_{\text {area }}} \\
&-\left[1-\frac{\pi\left(\sigma^{2}+\mu_{0}^{2}\right)}{S_{\text {area }}}\right]^{n} \geq \frac{\pi r^{2}}{S_{\text {area }}}-1 \\
&-\left[1-\frac{\pi\left(\sigma^{2}+\mu_{0}^{2}\right)}{S_{\text {area }}}\right]^{n} \geq\left[1-\frac{\pi r^{2}}{S_{\text {area }}}\right] \\
& {\left[1-\frac{\pi\left(\sigma^{2}+\mu_{0}^{2}\right)}{S_{\text {area }}}\right]^{n} \leq 1-\frac{\pi r^{2}}{S_{\text {area }}} } \\
& \log \left[1-\frac{\pi\left(\sigma^{2}+\mu_{0}^{2}\right)}{S_{\text {area }}}\right]^{n} \leq \log \left[1-\frac{\pi r^{2}}{S_{\text {area }}}\right]
\end{aligned}
$$




$$
\begin{aligned}
\mathrm{n} \log \left[1-\frac{\pi\left(\sigma^{2}+\mu_{0}^{2}\right)}{S_{\text {area }}}\right] & \leq \log \left[1-\frac{\pi r^{2}}{S_{\text {area }}}\right] \\
n & \leq \frac{\log \left(1-\left(\frac{\pi r^{2}}{S_{\text {area }}}\right)\right)}{\log \left(1-\left(\frac{\pi\left(\sigma^{2}+\mu_{0}^{2}\right)}{S_{\text {area }}}\right)\right)}
\end{aligned}
$$

Hence minimum sensor nodes are required for effective coverage of $S$ is

$$
\log \left(1-\left(\frac{\pi r^{2}}{S_{\text {area }}}\right)\right) / \log \left(1-\left(\frac{\pi\left(\sigma^{2}+\mu_{0}^{2}\right)}{S_{\text {area }}}\right)\right)
$$

\section{Basic Idea of Redundancy}

\subsection{Euclidean Distance}

\section{Case (i)}

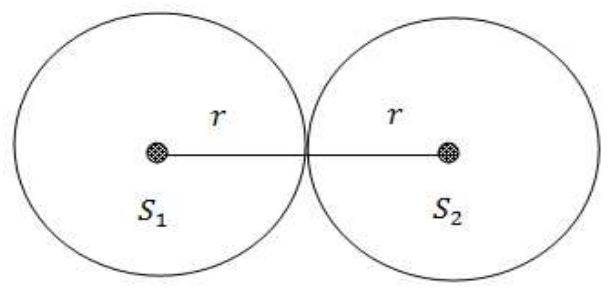

If $\left(x_{1}, y_{1}\right)$ and $\left(x_{2}, y_{2}\right)$ are the harmonize values of the sensor nodes $S_{1}, S_{2}$ then

$d\left(S_{1}, S_{2}\right)=\sqrt{\left(x_{1}-x_{2}\right)^{2}+\left(y_{1}-y_{2}\right)^{2}} \leq 2 r$ where $d$ is the Euclidean distance and $r$ is the radius of sensor nodes.

Case (ii) $r_{1}-r_{2}<d\left(S_{1}, S_{2}\right)<r_{1}+r_{2}$

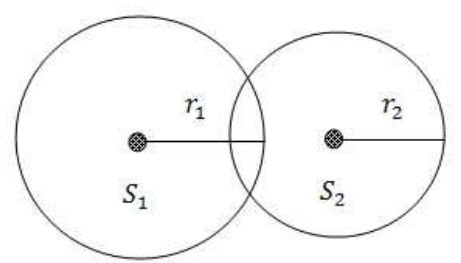

Case (iii) $d\left(S, S_{2}\right)>r_{1}+r_{2}$ 


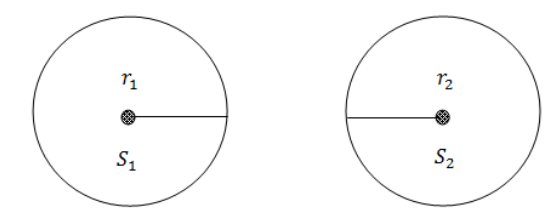

Case (iv) $d\left(S, S_{2}\right)=r_{1}+r_{2}$

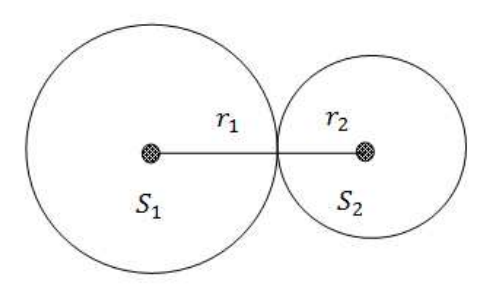

Case (v) $d\left(S, S_{2}\right)<r_{1}-r_{2}$

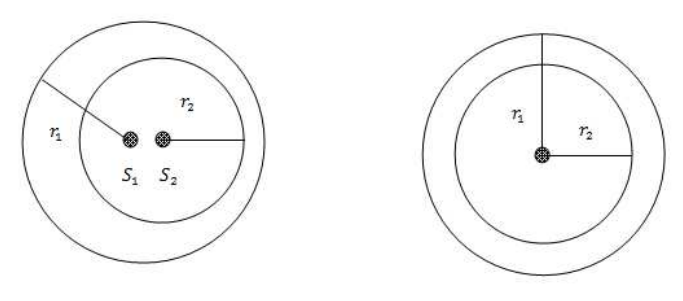

\subsection{Redundancy nodes}

If $S_{i}$ and $S_{j}$ are the surrounding nodes to one another in which the Euclidean distance between sensors nodes is smaller than the captious entry $i$ is called redundancy nodes.

\subsection{Redundant Coverage}

A certain rate of the sensing area of the redundancy nodes is called the redundant coverage.

\subsection{Determination of Redundancy Coverage}

Initially, the process of deployment the sensor nodes is emitted in $\mathrm{S}$ at randomly and in maximum frequency. So there exists the large number of nodes with redundancy in kicking around the observing spot. The increasing number of nodes can cause decreasing the throughout, 
network congestion and consuming high energy. So reducing the redundancy is more important to wireless sensor network.

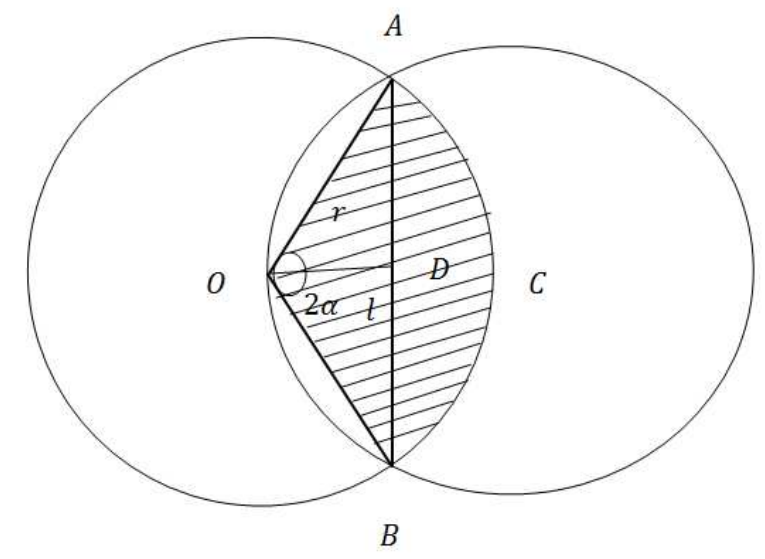

Fig: 5 Representation of node - joint coverage

Assume that the $n$ redundant surrounding nodes of the sensor node are distributed uniformly. Since the communication radius of sensor nodes is $\lambda r$ where $\lambda$ is the coefficient of proportion.

Let the distance between $O$ and $D$ is a random variable $l$ then $f(l)=\frac{l}{\pi r^{2}}, 0 \leq l \leq \lambda r$

In fig $4, \angle A O D=\alpha=\frac{\pi}{3}$

Area of the shaded region $=\frac{1}{3} \pi r^{2}$

Area of the $\triangle O A B=\frac{1}{2} r^{2} \sin 2 \alpha$

Area of $A C B=\frac{1}{3} \pi r^{2}-\frac{1}{2} r^{2} \sin 2 \alpha$

$$
\begin{aligned}
& =\left(\frac{1}{3}-\frac{1}{2} \sin 2 \alpha\right) r^{2} \\
& =\left(\alpha-\frac{1}{2} \sin 2 \alpha\right) r^{2}
\end{aligned}
$$

Area of $A C B=\frac{1}{2}(2 \alpha-\sin 2 \alpha) r^{2}$ 
Area of Intersection of circles $=2$ Area of $A C B$

Area $=(2 \alpha-\sin 2 \alpha) r^{2}$

Consider $l=r \cos \alpha, d l=-r \sin \alpha d \alpha$ and $\alpha \in\left[\pi / 2, \cos ^{-1} \lambda\right]$

Let $P$ be the redundant coverage then

$P=\int$ Area.$f(l) d l$

$=\int_{\pi / 2}^{\cos ^{-1} \lambda}(2 \alpha-\sin 2 \alpha) r^{2} \cdot \frac{l}{\pi r^{2}} d l$

$=\frac{1}{\pi} \int_{\pi / 2}^{\cos ^{-1} \lambda}-(2 \alpha-\sin 2 \alpha) r^{2} . \sin \alpha \cos \alpha d \alpha$

$=\frac{r^{2}}{2 \pi} \int_{\cos ^{-1} \lambda}^{\pi / 2}(2 \alpha-\sin 2 \alpha) \sin 2 \alpha d \alpha$

$=\frac{r^{2}}{2 \pi}\left[2 \int_{\cos ^{-1} \lambda}^{\pi / 2} \alpha \sin 2 \alpha d \alpha-\int_{\cos ^{-1} \lambda}^{\pi / 2} \sin ^{2} 2 \alpha d \alpha\right]$

$=\frac{r^{2}}{2 \pi}\left[\frac{-2 \alpha \cos 2 \alpha+\sin 2 \alpha}{2}-\frac{\alpha}{2}+\frac{\sin 4 \alpha}{8}\right]_{\cos ^{-1} \lambda}^{\pi / 2}$

$=\frac{r^{2}}{2 \pi}\left[\frac{\pi}{4}+\cos ^{-1} \lambda \cos \left(2 \cos ^{-1} \lambda\right)-\frac{\sin \left(2 \cos ^{-1} \lambda\right)}{2}+\frac{\cos ^{-1} \lambda}{2}+\frac{\sin \left(4 \cos ^{-1} \lambda\right)}{8}\right]$

$=\frac{r^{2}}{2 \pi}\left[\frac{\pi}{4}+2 \lambda^{2} \cos ^{-1} \lambda-\cos ^{-1} \lambda-\lambda \sqrt{1-\lambda^{2}}+\frac{1}{2} \cos ^{-1} \lambda-\lambda^{3} \sqrt{1-\lambda^{2}}+\frac{1}{2} \lambda \sqrt{1-\lambda^{2}}\right]$

$P=\frac{r^{2}}{2 \pi}\left[\frac{\pi}{4}+2 \lambda^{2} \cos ^{-1} \lambda-\frac{1}{2} \cos ^{-1} \lambda-\frac{\lambda}{2} \sqrt{1-\lambda^{2}}-\lambda^{3} \sqrt{1-\lambda^{2}}\right]$

If the redundant surrounding nodes covered by any sensor node then the redundant coverage $P_{\alpha}$

$$
\begin{aligned}
P_{\alpha} & =\frac{P}{\pi r^{2}} \\
& =\frac{1}{2 \pi^{2}}\left[\frac{\pi}{4}+\frac{1}{2}\left(4 \lambda^{2}-1\right) \cos ^{-1} \lambda-\frac{\lambda}{2} \sqrt{1-\lambda^{2}}-\lambda^{3} \sqrt{1-\lambda^{2}}\right]
\end{aligned}
$$

For $n$ communication nodes, by (2)

$P(n)=1-\left\{1-\frac{1}{2 \pi^{2}}\left[\frac{\pi}{4}+\frac{1}{2}\left(4 \lambda^{2}-1\right) \cos ^{-1} \lambda-\frac{\lambda}{2} \sqrt{1-\lambda^{2}}\left(1+2 \lambda^{2}\right)\right]\right\}^{n}$ 


\section{Conclusion}

With the aim of resolving the adversity of the coverage for wireless sensor network, we have produced a mathematical solution procedure of flawless ratio of coverage for three-sensor node configuration. Also, the solution procedures are explained for finding the estimated value of quality of coverage and requirement of least sensor nodes for effective coverage. Various basic terms are also part of the discussion. Finally, redundancy coverage is also determined for $n$ communication nodes. Also, this research investigation may be extended for boundary layers and roaming sensor nodes in the near future.

\section{Declaration}

- Funding - The author did not receive support from any organization for the submitted work.

- Conflicts of Interest - The author has no relevant financial or non-financial interests to disclose.

- Availability of Data \& Material - The author hereby declare that no specific data sets are utilized in the proposed work.

- Code Availability - Since, future works are based on the custom codes developed in this work, the code may not be available from the author.

- Author's contribution - The author is solely responsible for the experimental works conducted in this paper, drafting of the paper and presentation of all the sections

\section{References}

1. J.A. Bondy, U.S.R. Murty. Graph Theory with Applications, North-Holland, Amsterdam (1976).

2. Zhu KN, Zhu RB, Nii H, et al. PaperIO: a 3D interface towards the internet of embedded paper-craft. IEICE T Inf Syst 2014; E97-D(10): 2597-2605.

3. Derr K and Manic M. Wireless sensor network configuration-part II: adaptive coverage for decentralized algorithms. IEEE T Ind Inform 2013; 9(3): 1728-1738.

4. Zhu RB, Ma MD, Zhang Y, et al. Collaborative wireless sensor networks and applications. Int J Distrib Sens Netw 2015; 11: 1-2.

5. Adulyasas A, Sun ZL and Wang N. Connected coverage optimization for sensor scheduling in wireless sensor networks. IEEE Sens J 2015; 15(7): 3877-3892. 
6. Liao ZF, Wang JX, Zhang SG, et al. Minimizing movement for target coverage and network connectivity in mobile sensor networks. IEEE T Parallel Distrib Syst 2015; 26(7): 1971-1983.

7. Sun ZY, Wang HZ, Wu WG, et al. ECAPM: an enhanced coverage algorithm in wireless sensor networks based on probability model. Int J Distrib Sens Netw 2015; 2015(1): 111.

8. Mini S, Siba KU and Samrat LS. Sensor deployment and scheduling for target coverage problem in wireless sensor networks. IEEE Sens J 2014; 14(3): 636-644.

9. Zhao CJ, Wu HR, Liu Q, et al. Optimization strategy on coverage control in wireless sensor network based on Voronoi. J Commun 2013; 28(1): 36-43.

10. Hanid M, Kaveh M, Amir GA, et al. Distributed deployment algorithms for improved coverage in a network of wireless mobile sensors. IEEE T Ind Inform 2014; 10(1): 163174.

11. Zeyu Sun, Chuanfeng Li, Xiaofei Xing, Huihui wang, Ben Yan and Xuelun Li. k-degree coverage algorithm based on optimization nodes deployment in ireless sensor networks, International Journal of distributed sensor networks 2017; Vol. 13(2): 1-16.

12. Rasmi Ranjan Patra, Prashanta Kumar Patra. Analysis of k-coverage in ireless sensor networks, International Journal of Advanced Computer Science and Applications 2011; Vol. 2(9): 91-96.

13. Ming Liu, Jiannong Cao et al. Coverage analysis for ireless sensor networks, X. Jia, J. Wu and Y. He (Eds.) 2005; 711-720.

14. Xiaole Bai, Santhosh Kumar et al. Deploying wireless sensors to achieve both coverage and connectivity, MobiHoc 2006, Florence, Italy: 131-147.

15. Xiaole Bai,Zigui Yun et al. Optimal patterns for four-Connectivity and full coverage in wireless sensor networks, IEEE Transactions on Mobile Computing 2010: Vol 9 (3) : 435-448.

16. Tabirca. T, Yang L. T and Tabirca. S. Smallest number of sensors for k- covering, Int J Comput Commun 2013: Issn 1841-9836, Vol. 8 :312-319.

17. Ammari, H. M., Das, S.K, Clustering based minimum energy ireless m-connected, kcovered sensor networks, Proc. Of 2008 ESWN Sensor, LNCS 4913, 1-6. 